\title{
Optimal Transmission Probability in Binary Molecular Communication
}

\author{
Baris Atakan
}

\begin{abstract}
Molecular communication (MC) is a promising nanoscale communication paradigm that enables nanomachines to share information by using messenger molecules. In this paper, an expression for the achievable rate in MC is first given. Then, using this expression, an optimal transmission probability is developed to maximize the MC rate. Numerical results show that the MC rate is time-dependent and the molecules freely wandering in the medium negatively affect the $\mathrm{MC}$ performance. However, the proposed optimal transmission probability is shown to maximize the MC rate.
\end{abstract}

Index Terms-Molecular communications, nanonetworks, achievable rate, optimal transmission.

\section{INTRODUCTION}

$\mathbf{R}$ APID development in nano and biotechnology currently paves the way for the fabrication of the nanoscale machines called NanoMachines (NMs). NMs have great potential to provide many promising applications in medicine and industry. However, the realization of these applications is severely prone to uncontrollability and unstability problems due to very tiny sizes and unknown physics of NMs. Nevertheless, these problems can be addressed by coordinating NMs via a nanoscale communication. This forms a nanonetwork of NMs and can coordinate different NM populations to reach a sophisticated behavior and to increase the number of design possibilities. Molecular communication (MC) in which molecules are used to share information is one of the most promising paradigms for the realization of nanonetworks [1].

The nanonetworks with MC are conceptualized in [1] and [2]. Besides these initial works, achievable MC rate is investigated [3]-[7]. The molecular noise in the $\mathrm{MC}$ channel is also broadly investigated and modeled in [8]. A rigorous stochastic analysis of MC and a noise model for $\mathrm{MC}$ are also presented in [9]. The deterministic capacity of molecular information flow in a nanonetwork is explored and the necessity of networking techniques for the realization of future nanonetworks is also discussed in [10]. There are also more recent works on the capacity of molecular communication. In [11], communication capacity is comprehensively investigated for a diffusion-based molecular communication system by taking into account the channel memory and molecular noise. A channel model and capacity analysis are also given in [12] for the case in which the messenger molecules have 1-D motion and they may degrade. In [13], a molecular relay channel is analyzed to find out its communication capacity. The performance of the active molecular communication in microchannels is also

Manuscript received February 1, 2013. The associate editor coordinating the review of this letter and approving it for publication was M. Matthaiou.

The author is with the Department of Electrical and Electronics Engineering, Izmir Institute of Technology, Izmir, 35430, Turkey (e-mail: barisatakan@iyte.edu.tr).

Digital Object Identifier 10.1109/LCOMM.2013.041813.130255

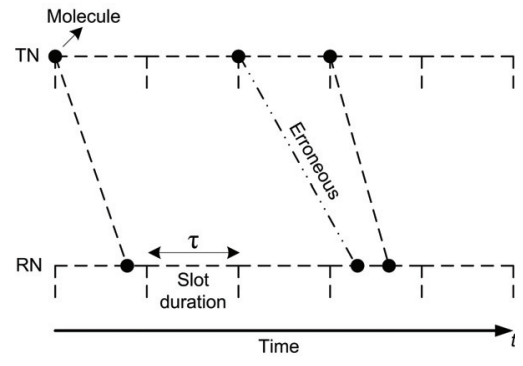

Fig. 1. A timing diagram of the MC between $\mathrm{TN}$ and $\mathrm{RN}$.

explored in [14]. Furthermore, the recent survey papers [15][17] extensively overview the current literature of molecular communication.

In this paper, an expression is derived for MC rate. Then, based on this expression an optimal transmission probability is derived to ensure the maximum achievable MC rate. Next, a MC model and a rate expression for MC are introduced in Section II. An optimal transmission probability is derived in Section III. Finally, numerical results and concluding remarks are presented in Section IV and V.

\section{Binary Molecular Communication Model}

In this paper, a binary $\mathrm{MC}$ scheme is considered. In order to transmit bit 1 , the Transmitter Nanomachine (TN) emits a molecule at the beginning of the slot duration ${ }^{1}$, i.e., $\tau$. The bit 1 is assumed to be successfully delivered if the Receiver Nanomachine (RN) receives the molecule during $\tau$. For the transmission of bit 0 , TN emits no molecule and it is assumed to be successfully delivered if RN does not receive any molecule during $\tau$. Otherwise, the current transmission attempt becomes unsuccessful. In Fig. 1, a timing diagram for the defined binary MC scheme is illustrated. The movement of molecules is assumed to be governed by a Brownian motion. Then, the delay $t$ experienced by any molecule to reach $\mathrm{RN}$ obeys the following probability density function [19].

$$
f(t)=\frac{d}{\sqrt{4 \pi D t^{3}}} e^{-\frac{d^{2}}{4 D t}}, \quad t>0
$$

where $D$ is the diffusion coefficient of the molecules and $d$ is the Euclidean distance between TN and RN. Notice that $f(t)$ characterizes the delay for one dimensional motion of molecules. However, all analyses given in the rest of the paper are directly applicable to two and three dimensional motion with appropriate probability density functions. The cumulative

\footnotetext{
${ }^{1}$ Here, the nanomachines are assumed to synchronize with each other to emit and receive each molecule within the fixed duration time slots. Note that synchronization of nanomachines has been already investigated in the literature. For example, in [18], the oscillatory behavior of individual cells can be synchronized via inter-cell signaling.
} 
distribution function associated with the density function $f(t)$ in (1), i.e., $F(t)$, can be given as

$$
F(t)=\operatorname{Erfc}\left(\frac{d}{2 \sqrt{D t}}\right), \quad t>0 .
$$

Let assume that TN transmits 1 at slot $n$ with the probability $\beta_{n}$ or transmits 0 with the probability $\left(1-\beta_{n}\right)$. Hence, the channel input at slot $n$, i.e., $X_{n}$, can be characterized as $X_{n} \sim \operatorname{Bernoulli}\left(\beta_{n}\right), \forall n \in\{1,2, \ldots\}$. If the molecule emitted at slot $n$ is just taken into account, the following probabilities can be derived. For the case of $X_{n}=1$, the probability that RN successfully receives 1 , i.e., $\alpha_{n}$, can be given as $\alpha_{n}=\beta_{n} F(\tau)$. However, the probability that the emitted molecule fails to reach $\mathrm{RN}$ and 1 cannot be successfully received within slot $n$, i.e., $\xi_{n}$, is $\xi_{n}=\beta_{n}(1-F(\tau))$. In this case, RN erroneously receives 0 instead of 1 . Furthermore, if TN emits no molecule at slot $n$, which means $X_{n}=0$, the probability that RN successfully receives 0 , i.e., $\zeta_{n}$, can be expressed as $\zeta_{n}=\left(1-\beta_{n}\right)$. Consequently, if the molecule emitted at slot $n$ is just taken into account, RN receives 1 with $\alpha_{n}$ and receives 0 with $\xi_{n}+\zeta_{n}=\left(1-\alpha_{n}\right)$ during slot $n$. This can be also characterized by a Bernoulli random variable, i.e., $G_{n} \sim \operatorname{Bernoulli}\left(\alpha_{n}\right)$.

In addition to the molecule emitted at slot $n$, RN may also receive the molecules that have been transmitted for the previous $n-1$ slot duration and have not been received by $\mathrm{RN}$. Suppose that a molecule, that is emitted at slot $k$ with probability $\beta_{k}$ and has not been received by RN. Note that $k \in\{1,2, \ldots, n-1\}$. Clearly, this molecule may be received by RN at slot $n$ since every emitted molecule continuously diffuses in the medium until it is received by RN. In such a case, the probability that the molecule, emitted at slot $k$, is received at slot $n$, i.e., $\lambda_{n k}$, can be given as

$$
\lambda_{n k}=\beta_{k}[F((n-k+1) \tau)-F((n-k) \tau)], \quad k<n
$$

Notice that $\lambda_{n k}$ is only available for $k<n$. Furthermore, the probability that the molecule emitted at slot $k$ is not received by $\mathrm{RN}$ during slot $n$ is $\left(1-\lambda_{n k}\right)$. In fact, such a late molecule can be considered as a noise of the channel and its effect on the channel, i.e., $\Gamma_{n, k}$, can be characterized by a Bernoulli random variable, that is, $\Gamma_{n, k} \sim \operatorname{Bernoulli}\left(\lambda_{n k}\right)$. Similar to slot $k$, each noise term coming from previous $(n-1)$ slots can be modeled as a Bernoulli random variable, i.e., $\Gamma_{n, k} \sim$ Bernoulli $\left(\lambda_{n k}\right), \forall k \in\{1,2, \ldots, n-1\}$. Hence, by adding all of these noise terms, total noise at slot $n$, i.e., $Z_{n}$, can be expressed as

$$
Z_{n}=\sum_{k=1}^{n-1} \Gamma_{n, k}
$$

Here, note that each $\Gamma_{n, k}$ is independent of each other since each of them depends on a different channel transmission probability $\beta_{k} k \in\{1, \ldots, n-1\}$. Furthermore, $\Gamma_{n, k}, \forall k$ are independent from $G_{n}$ since $G_{n}$ only depends on $\beta_{n}$ while $\Gamma_{n, k}, \forall k$ do not depend on $\beta_{n}$. By considering $G_{n} \sim$ $\operatorname{Bernoulli}\left(\beta_{n} F(\tau)\right)$ and $Z_{n}$, the channel output at slot $n$, i.e., $Y_{n}$, can be given as $Y_{n}=G_{n}+Z_{n}$. Here, the channel output, $Y_{n}$, and noise, $Z_{n}$, are the sum of Bernoulli random variables with ranges $\{0,1,2, \ldots, n\}$ and $\{0,1,2, \ldots, n-1\}$, respectively. Thus, they can be characterized by two PoissonBinomial random variables. Using the probability $\lambda_{n k}$ in (3), the Probability Mass Functions (PMFs) of $Y_{n}$ and $Z_{n}$ can be respectively given as

$$
\begin{aligned}
p_{i} & =\sum_{A \in \mathcal{S}_{i}} \prod_{k \in A} \lambda_{n k} \prod_{j \in A^{c}}\left(1-\lambda_{n j}\right) \\
q_{i} & =\sum_{B \in \mathcal{T}_{i}} \prod_{l \in B} \lambda_{n l} \prod_{m \in B^{c}}\left(1-\lambda_{n m}\right)
\end{aligned}
$$

where $p_{i}$ and $q_{i}$ are the point probabilities of $Y_{n}$ and $Z_{n}$, that is, $\operatorname{Pr}\left(Y_{n}=i\right)=p_{i}, i \in\{0,1, \ldots, n\}$ and $\operatorname{Pr}\left(Z_{n}=\right.$ $i)=q_{i}, i \in\{0,1, \ldots, n-1\} . \mathcal{S}_{i}$ and $\mathcal{T}_{i}$ are the sets that include all subsets of $i$ integers that can be selected from $\{1,2, \ldots, n\}$ and $\{1,2, \ldots, n-1\}$, respectively. For example, if $n=3$, then $i$ is selected from $\{1,2,3\}$ to form $\mathcal{S}_{i}$ and selected from $\{1,2\}$ to form $\mathcal{T}_{i}$. In this case, $\mathcal{S}_{2}$ and $\mathcal{T}_{2}$ can be given as $\mathcal{S}_{2}=\{\{1,2\},\{1,3\},\{2,3\}\}$ and $\mathcal{T}_{2}=\{\{1,2\}\}$. $A$ denotes the elements of $\mathcal{S}_{i}$ and $A^{c}$ is the complement of $A$, i.e., $A^{c}=\{1,2, \ldots, n\} \backslash A$. Furthermore, $B$ denotes an element of $\mathcal{T}_{i}$ and $B^{c}$ is the complement of $B$.

The mutual information between the channel input $X_{n} \sim$ Bernoulli $\left(\beta_{n}\right)$ and the output $Y_{n}$ with the PMF given in (5) can be expressed as

$$
\begin{aligned}
I\left(X_{n} ; Y_{n}\right) & =H\left(Y_{n}\right)-H\left(Y_{n} \mid X_{n}\right) \\
& =H\left(G_{n}+Z_{n}\right)-H\left(G_{n}+Z_{n} \mid X_{n}\right)
\end{aligned}
$$

Since $X_{n} \sim \operatorname{Bernoulli}\left(\beta_{n}\right)$ and $G_{n} \sim \operatorname{Bernoulli}\left(\beta_{n} F(\tau)\right)$ and $F(\tau)$ is a constant probability, $G_{n}$ can be determined if $X_{n}$ is given. Furthermore, $X_{n}$ and $Z_{n}$ are independent. Thus, $H\left(G_{n} \mid X_{n}\right)=0$ and $H\left(Z_{n} \mid X_{n}\right)=H\left(Z_{n}\right)$ and using $p_{i}$ and $q_{i}$ in (5) and (6), $I\left(X_{n} ; Y_{n}\right)$ can be written as

$$
\begin{aligned}
I\left(X_{n} ; Y_{n}\right) & =H\left(Y_{n}\right)-H\left(Z_{n}\right) \\
& =-\sum_{i=0}^{n} p_{i} \log p_{i}+\sum_{i=0}^{n-1} q_{i} \log q_{i}
\end{aligned}
$$

Note that each noise term in (4) can be considered as the effect of channel memory. These effects are summarized in $Z_{n}$ to obtain an approximation for the maximum achievable MC rate. Hence, $I\left(X_{n} ; Y_{n}\right)$ can be considered as $\frac{1}{n} I\left(X_{1}, X_{2}, \ldots, X_{n} ; Y_{1}, Y_{2}, \ldots, Y_{n}\right)$. Next, a close-form expression is derived for the optimal value of $\beta_{n}$ which maximizes $I\left(X_{n} ; Y_{n}\right)$ to obtain the maximum achievable rate during the slot $n$.

\section{Optimal Transmission Probability in MC}

In this section, a tight approximation for the optimal $\beta_{n}$ is provided to maximize $I\left(X_{n} ; Y_{n}\right)$. To this end, using the statistical dependence between $p_{i}$ and $q_{i}$ given in (5) and (6), $p_{i}$ can be first expressed as a function of $q_{i}$ and $\alpha_{n}$ as follows.

$$
\begin{aligned}
p_{i} & =\alpha_{n} q_{i-1}+\left(1-\alpha_{n}\right) q_{i} \\
& =q_{i}+\alpha_{n}\left(q_{i-1}-q_{i}\right) \\
& =q_{i}+\beta_{n} F(\tau)\left(q_{i-1}-q_{i}\right) \\
& =q_{i}+\beta_{n} F(\tau) r_{i}
\end{aligned}
$$


where $r_{i}=q_{i-1}-q_{i}$. By substituting $p_{i}=q_{i}+\beta_{n} F(\tau) r_{i}$ into $I_{n}\left(X_{n} ; Y_{n}\right), \frac{\partial I_{n}}{\partial \beta_{n}}$ can be written as

$$
\frac{\partial I_{n}}{\partial \beta_{n}}=-\sum_{i=0}^{n} F(\tau) r_{i} \log \left[q_{i}+\beta_{n} F(\tau) r_{i}\right]-\sum_{i=0}^{n} F(\tau) r_{i}
$$

Note that $I_{n}$ is hereafter used to denote $I\left(X_{n} ; Y_{n}\right)$. Since $q_{-1}$ and $q_{n}$ are equal to $0, \sum_{i=0}^{n} F(\tau) r_{i}=F(\tau)\left(q_{-1}-q_{0}+q_{0}-\right.$ $\left.q_{1}+q_{1}-q_{2}+\ldots-q_{n-1}+q_{n-1}-q_{n}\right)=0$. Then, (10) can be given as

$$
\begin{array}{r}
\frac{\partial I_{n}}{\partial \beta_{n}}=-F(\tau) r_{0} \log \left[q_{0}+\beta_{n} F(\tau) r_{0}\right]- \\
-\sum_{i=1}^{n} F(\tau) r_{i} \log \left[\left[1+\frac{q_{i}}{\beta_{n} F(\tau) r_{i}}\right] \beta_{n} F(\tau) r_{i}\right]
\end{array}
$$

For the solution of $\frac{\partial I_{n}}{\partial \beta_{n}}=0$, the approximation, $\log (1+x) \approx x$ for $|x|<0.1$, can be applied to $\log \left[1+\frac{q_{i}}{\beta_{n} F(\tau) r_{i}}\right]$ in (11) for $\left|\frac{q_{i}}{\beta_{n} F(\tau) r_{i}}\right|<0.1 \forall i$ and this yields

$$
\begin{array}{r}
\frac{\partial I_{n}}{\partial \beta_{n}} \approx-F(\tau) r_{0} \log \left[q_{0}+\beta_{n} F(\tau) r_{0}\right]- \\
-\sum_{i=1}^{n} F(\tau) r_{i}\left[\log \left[F(\tau) r_{i}\right]+\log \left[\beta_{n}\right]\right]-\sum_{i=1}^{n} \frac{q_{i}}{\beta_{n}}
\end{array}
$$

Due to $\sum_{i=1}^{n} r_{i}=q_{0}, \sum_{i=1}^{n} q_{i}=1-q_{0}$, and $r_{0}=-q_{0}$, (12) can be also modified as

$$
\begin{gathered}
\frac{\partial I_{n}}{\partial \beta_{n}} \approx-F(\tau) q_{0}\left[\log \left(1+\frac{\beta_{n}+\beta_{n} F(\tau)-1}{1-\beta_{n} F(\tau)}\right)\right]- \\
\frac{\left(1-q_{0}\right)}{\beta_{n}}-\sum_{i=1}^{n} F(\tau) r_{i} \log \left[F(\tau) r_{i}\right]-F(\tau) q_{0} \log \left[\frac{1}{q_{0}}\right]
\end{gathered}
$$

In (13), $\log \left(1+\frac{\beta_{n}+\beta_{n} F(\tau)-1}{1-\beta_{n} F(\tau)}\right)$ can be approximated as $\frac{\beta_{n}+\beta_{n} F(\tau)-1}{1-\beta_{n} F(\tau)}$ for $\left|\frac{\beta_{n}+\beta_{n} F(\tau)-1}{1-\beta_{n} F(\tau)}\right|<0.1$ and substituting this approximation into (13),

$$
\begin{gathered}
\frac{\partial I_{n}}{\partial \beta_{n}} \approx-F(\tau) q_{0}\left[\frac{\beta_{n}+\beta_{n} F(\tau)-1}{1-\beta_{n} F(\tau)}\right]-\frac{\left(1-q_{0}\right)}{\beta_{n}} \\
-\sum_{i=1}^{n} F(\tau) r_{i} \log \left[F(\tau) r_{i}\right]-F(\tau) q_{0} \log \left[\frac{1}{q_{0}}\right]
\end{gathered}
$$

can be obtained. Finally, solving $\frac{\partial I_{n}}{\partial \beta_{n}}=0$ in (14), the optimal $\beta_{n}$, i.e., $\widehat{\beta}_{n}$, can be given as in (18). In (18), $\delta$ is

$$
\delta=\sum_{i=1}^{n} F(\tau) r_{i} \log F(\tau) r_{i}-F(\tau) q_{0} \log \left(q_{0}\right)
$$

Since $\log (1+x) \approx x$ is tight for $|x|<0.1$, the tightness of the approximations in (12) and (14) also requires to satisfy the following two conditions, i.e., $c n d_{1}$ and $c n d_{2}$ :

$$
\begin{aligned}
& c n d_{1}=\left|\frac{q_{i}}{\beta_{n} F(\tau) r_{i}}\right|<0.1, \forall i \in\{1, \ldots, n-1\} \\
& c_{n}=\left|\frac{\beta_{n}+\beta_{n} F(\tau)-1}{1-\beta_{n} F(\tau)}\right|<0.1
\end{aligned}
$$

In Section IV, these conditions, $c n d_{1}$ and $c n d_{2}$, are numerically evaluated for the tightness of the approximations. As observed in (18), the optimal value of $\beta_{n}$, i.e. $\widehat{\beta}_{n}$, is a function of $F(\tau)$ and $q_{i}, i \in\{0, \ldots, n-1\}$. Hence, it is possible to

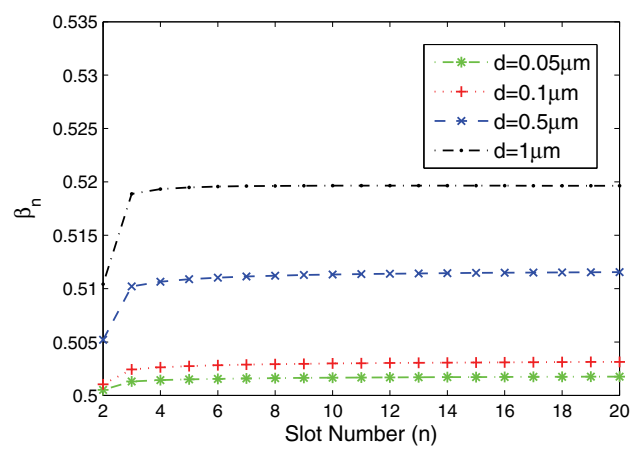

Fig. 2. $\widehat{\beta}_{n}$ with varying slot number for the different distance values, i.e., $d$, between $\mathrm{TN}$ and $\mathrm{RN}$.

iteratively compute $\widehat{\beta}_{n}$ for each slot $n$. For example, at slot 10 , $\widehat{\beta}_{10}$ can be computed by using $F(\tau)$ and $q_{i}, i=\{0, \ldots, 9\}$. For this, an arbitrary $\beta_{1}$ is initially selected, then, $\widehat{\beta}_{2}$ are found by computing $q_{0}$ and $q_{1}$ and substituting them into (18) and (15). Similarly, each $\widehat{\beta}_{n}, n \in\{2, \ldots\}$ can be consecutively computed by using $\beta_{1}, \widehat{\beta}_{2}, \ldots, \widehat{\beta}_{n-1}$. Next, we present the numerical results on the maximum MC rate and $\widehat{\beta}_{n}$.

\section{NumericAl Results}

In this section, the numerical results on the optimal transmission probability, $\widehat{\beta}_{n}$, in (18) and the maximum achievable MC rate obtained by using $\widehat{\beta}_{n}$ are presented for varying system parameters. TN is assumed to emit molecules by starting from slot 1 . Furthermore, $\beta_{1}$ is set to $\beta_{1}=0.3$ and $\tau$ is set to $\tau=0.5 \mathrm{~s}$ throughout the numerical evaluations. In Fig. 2, $\widehat{\beta}_{n}$ is shown as the slot number changes from 1 to 20 for different distance values, i.e., $d$, between TN and RN. For all $d$ values ranging from $d=0.05 \mu \mathrm{m}$ to $d=1 \mu \mathrm{m}, \widehat{\beta}_{n}$ slightly increases with $d$ and quickly converges to the optimal values rather than oscillating around some specific values. This can make the determination of the efficient channel codes feasible. The convergent $\widehat{\beta}_{n}$ is used to determine the average number of bits 1 in the code words to ensure high MC rate. For the same setting used in Fig. 2, maximum achievable MC rate, i.e., $C_{n}$, is shown in Fig. 3. Note that $C_{n}$ values in Fig. 3 are computed by using $\widehat{\beta}_{n}$ values in Fig. 2 . The maximum achievable MC rate grows from 0.61 to 0.69 nats/trans. or from 0.88 to 0.993 bits/trans. at slot 20 as TN and RN become close to each other. This reveals that the optimal transmission probability provides a very high molecular communication rate up to $0.993 \mathrm{bits} /$ trans.. Furthermore, $C_{n}$ reduces as the slot number proceeds and this reduction becomes higher as $d$ increases because the diffusing molecules negatively affect the MC performance. However, this decrease in $C_{n}$ requires a rigorous convergence analysis to determine the lower-bound of the rate. Furthermore, in order to show the tightness of the $\widehat{\beta}_{n}$ computation in Fig. 2, the conditions $c n d_{1}$ and $c n d_{2}$ are shown in Fig. 4 and 5, respectively. Since $\left|c n d_{1}\right|<0.1$ and $\left|c n d_{2}\right|<0.1$ can be always satisfied as observed in the figures, the computed $\widehat{\beta}_{n}$ values are the tight approximations to the optimal values of the channel transmission probability $\beta_{n}$. 


$$
\widehat{\beta}_{n} \approx \frac{-\delta+F(\tau)-\sqrt{[\delta-F(\tau)]^{2}-4\left(1-q_{0}\right)\left[-\delta F(\tau)+F(\tau) q_{0}+F(\tau)^{2} q_{0}\right]}}{2\left[-\delta F(\tau)+F(\tau) q_{0}+F(\tau)^{2} q_{0}\right]}
$$

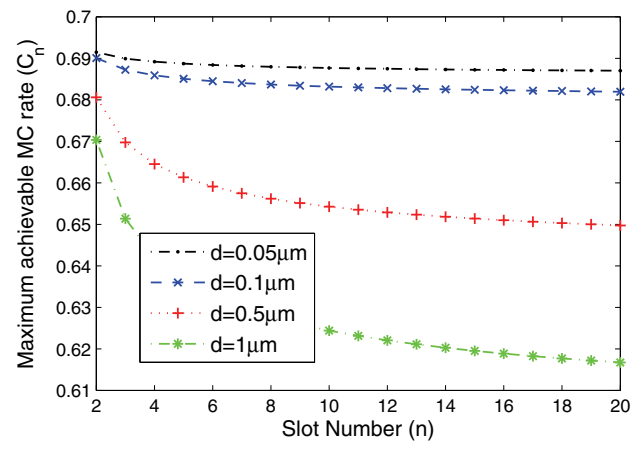

Fig. 3. The maximum achievable $\mathrm{MC}$ rate, i.e., $C_{n}$, with varying slot number for the different values of $d$.

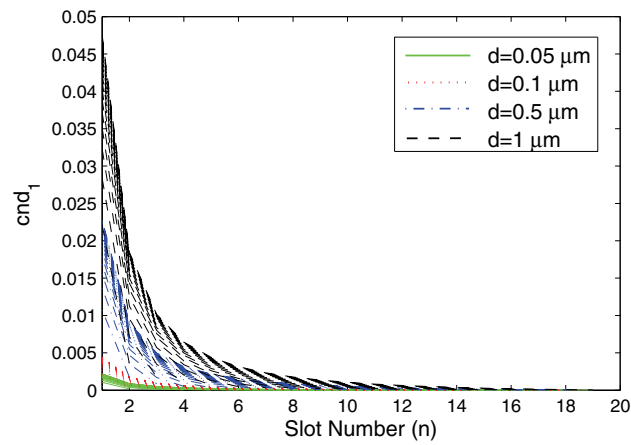

Fig. 4. $\quad c n d_{1}$ with varying slot number for the different $d$ values.

\section{Conclusion}

In this paper, a rate expression for molecular communication are first developed. Then, an optimal transmission probability is derived to reach the maximum achievable MC rate. The results reveal that the freely wandering molecules negatively affect the MC rate. However, the proposed optimal transmission probability can maximize the MC rate by taking into account all emitted molecules.

\section{REFERENCES}

[1] S. Hiyama, et al., "Molecular communication," in Proc. 2005 NSTI Nanotech.

[2] I. F. Akyildiz, F. Brunetti, C. Blazquez, "NanoNetworking: a new communication paradigm," Computer Networks J., vol. 52, no. 12, pp. 2260-2279, Aug. 2008.

[3] B. Atakan and O. B. Akan, "On channel capacity and error compensation in molecular communication," Springer Trans. Computational System Biology, vol. 10, pp. 59-80, Feb. 2008.

[4] D. Arifler, "Capacity analysis of a diffusion-based short-range molecular nano-communication channel," Computer Networks, vol. 55, no. 6, pp. 1426-1434, Apr. 2011.

[5] M. Ş. Kuran, H. B. Yılmaz, T. Tuğcu, and B. Özerman, "Energy model for communication via diffusion in nanonetworks," Nano Commun. Networks, vol. 1, no. 2, pp. 86-95, June 2010.

[6] M. U. Mahfuz, D. Makrakis, and H. T. Mouftah, "On the characterization of binary concentration-encoded molecular communication in nanonetworks," Nano Commun. Networks, vol. 1, no. 4, pp. 289-300, 2010.

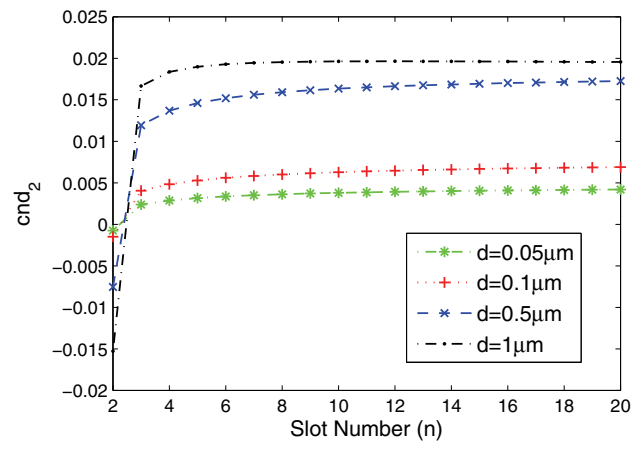

Fig. 5. $c n d_{2}$ with varying slot number for the different values of $d$.

[7] K. V. Srinivas, R. S. Adve, and A. W. Eckford, "Molecular communication in fluid media: the additive inverse Gaussian noise channel," IEEE Trans. Inf. Theory, vol. 58, no. 7, pp. 4678-4692, Jul. 2012.

[8] M. Pierobon and I. F. Akyildiz, "Diffusion-based noise analysis for molecular communication in nanonetworks," IEEE Trans. Signal Process., vol. 59, no. 6, pp. 2532-2547, June 2011.

[9] D. Miorandi, "A stochastic model for molecular communications," Nano Commun. Networks, vol. 2, no. 4, pp. 205-212, Dec. 2011.

[10] B. Atakan and O. B. Akan, "Deterministic capacity of information flow in molecular nanonetworks," Nano Commun. Networks, vol. 1, no. 1, pp. 31-42, Mar. 2010.

[11] M. Pierobon and I. F. Akyildiz, "Capacity of a diffusion-based molecular communication system with channel memory and molecular noise," IEEE Trans. Inf. Theory, vol. 59, no. 2, pp. 942-954, 2013.

[12] T. Nakano, O. Yutaka, and L. Jian-Qin, "Channel model and capacity analysis of molecular communication with Brownian motion," IEEE Commun. Lett., vol. 16, no. 6, pp. 797-800, 2012.

[13] T. Nakano and L. Jian-Qin, "Design and analysis of molecular relay channels: an information theoretic approach," IEEE Trans. Nanobioscience, vol. 9, no. 3, pp. 213-221, Sept. 2010.

[14] N. Farsad, A. W. Eckford, S. Hiyama, and Y. Moritani, "Information rates of active propagation in microchannel molecular communication," Lecture Notes of ICST, vol. 87, pp. 16-21, 2012.

[15] I. Llatser, A. Cabellos-Aparicio, and E. Alarcón, "Networking challenges and principles in diffusion-based molecular communication," IEEE Wireless Commun. Mag., vol. 19, no. 5, pp. 36-41, 2012.

[16] M. S. Kuran, T. Tugcu, and B. Ozerman, "Calcium signaling: overview and research directions of a molecular communication paradigm," IEEE Wireless Commun. Mag., vol. 19, no. 5, pp. 20-27, 2012.

[17] T. Nakano, et al., "Molecular communication and networking: opportunities and challenges," IEEE Trans. Nanobiosciences, vol. 11, no. 2, pp. 135-148, 2012.

[18] D. McMillen, N. Kopell, J. Hasty, and J. J. Collins, "Synchronizing genetic relaxation oscillators by intercell signaling," Proc. National Academy of Sciences of USA, vol. 99, pp. 679-684, 2002.

[19] I. Karatzas and S. E. Shreve, Brownian Motion and Stochastic Calculus. Springer, 1991. 